This is a post-peer-review, pre-copyedit version of an article published in Experimental Brain Research. The final authenticated version is available online at: https://doi.org/10.1007/s00221-021-06053-4 


\section{ERP evidence of age-related differences in emotional processing}

Roberta A. Allegretta ${ }^{1}$, Wesley Pyke², Giulia Galli

${ }^{1}$ Kingston University, Department of Psychology. Penrhyn Road, KT1 2EE Kingston Upon Thames. United Kingdom

${ }^{2}$ University of Kent, School of Psychology. Keynes College, CT2 7FH Canterbury. United Kingdom

* corresponding author: g.galli@kingston.ac.uk 


\section{ABSTRACT}

The present study used event-related potentials (ERPs) to examine differences in the temporal dynamics of emotion processing in young and older adults, with a specific focus on the positivity effect, that is, the preferential processing of positive over negative information. To this aim, we used a language paradigm that allowed us to investigate early ERP components as well as later components, namely the N400 and the Late Positive Complex (LPC). Young and older adults were presented with neutral sentence stems with positive, negative or neutral/semantically-incongruent critical word endings while their electrical brain activity was recorded. There were no effects of emotional valence on early ERP components. Instead, a positivity effect was evident in young adults indexed by reduced N400s for positive sentence endings. Perhaps due to reduced semantic processing abilities, older adults did not show any N400 effect. ERP effects in this group were evident at a later processing stage and took the form of larger LPCs for neutral/incongruent information. Overall, there was no effect of emotional valence on either the N400 or the LPC in older adults. Our data suggest that with age more effortful semantic processing may deplete resources for emotional processing. 


\section{INTRODUCTION}

Healthy ageing is characterised by a decline in a number of cognitive functions, including memory, attention and executive functions (Craik and Salthouse 2002). Anecdotal and empirical evidence, however, suggests that not all mental processes change for the worse with ageing. For instance, memory for facts, knowledge and procedures shows few agerelated changes (Luo and Craik 2008). Older adults also show higher levels of emotional well-being and a decrease in negative affect compared to younger adults (Carstensen et al. 2011). Indeed, one of the main characteristics of emotional processing in older age is a 'positivity effect' on cognition, which results from a preference for positive over negative information during cognitive processing (Mather and Carstensen 2003). For instance, older adults preferentially look toward faces displaying positive emotions (Mather and Carstensen 2003), especially when in a bad mood (Isaacowitz et al. 2008) and remember them better compared to faces displaying negative emotions (Charles, Mather and Carstensen 2003). Older adults also look less at negative parts of complex emotional visual scenes (Isaacowitz and Choi, 2011), especially when they are explicitly instructed to regulate their emotions (Noh, Lohani and Isaacowitz, 2011). Preferential processing of positive information was also demonstrated in decision-making (Löckenhoff and Carstensen 2007), working memory (Mikels et al. 2005) and autobiographical memory (Kennedy, Mather and Carstensen 2004) tasks. These findings have often been interpreted within the Socioemotional Selectivity Theory, which hypothesises that shorter time horizons may lead to a shift in emotionoriented goals and a prioritization of positively-valenced emotional information (Carstensen 2006). As a consequence, to achieve emotional wellbeing older adults may allocate more resources to material that optimizes emotion regulation (Urry and Gross 2010).

Neuroimaging methods can provide relevant insights into the brain mechanisms behind the positivity effect and of emotional processing more in general. Although the brain mechanisms of emotional processing have been widely examined in young adults, far less is known about how emotions are processed in the brain of older adults. In particular, one question that remains unanswered is whether an age-related difference exists in the 
temporal dynamics of emotion processing. With respect to the positivity effect for instance, older adults may show enhanced automatic processing of positive information at an early stage of processing, or age-related differences could emerge at later stages and be associated with enhanced meaning-based processing of positive information. Discerning between these two scenarios requires a technique that allows tracking in time of mental processes. Using eye-tracking Isaacowitz et al. (2009) found that older adults' gaze preference for happy faces emerged after approximately $500 \mathrm{~ms}$ from the onset of a face and increased linearly with time, suggesting that emotional processing in older adults is characterised by a preferential processing of positive information at a later stage of processing. Eye-tracking however only provides an indirect index of processing times in the brain. EEG instead records brain responses with excellent temporal resolution, thereby allowing insights into the temporal dynamics of emotion processing. In particular, eventrelated potentials (ERPs) are small voltage changes in the brain in response to events or stimuli.

Some ERP studies suggested that age-related differences in emotional processing can be evident in early ERP deflections that are generally associated with perceptual or attention processing. For instance, Hilimire et al. (2014) found that at frontal scalp sites, compared to their younger counterparts, older adults displayed larger positivities for happy faces as early as 130 ms from stimulus onset. Pollock et al. (2012) found that the P1, an ERP component related to early perceptual processing recorded from posterior electrodes, was larger in young compared to older adults in response to negative facial expressions. Age-related differences in emotion processing thus may start at a very early stage of processing. In the current investigation we reasoned that, if the positivity effect is based on a rapid, automatic preferential processing of positive information, we would observe modulations of early ERP deflections such as the P1, N1, N2 or P2. We further reasoned that these modulations would be larger for positively-valenced material in older than younger adults, in line with the notion of a positivity effect in ageing. 
An alternative hypothesis is that age-related differences in emotional processing emerge at later, meaning-based stage of processing. To test this hypothesis, we focused on the N400 component. The N400 is a negative-going waveform peaking at around $400 \mathrm{~ms}$ after stimulus onset with a centro-parietal distribution (Kutas and Hillyard 1980). The N400 is evoked by semantic anomalies or incongruences (e.g. "I drink my coffee with milk and socks", Kutas and Hillyard 1980) or by stimuli that are inconsistent with our views of the world (Van Berkum et al. 2009; Galli et al. 2017). In sequential presentation paradigms where a critical target word is presented following the presentation of contextual information (e.g., a preceding sentence stem), the N400 is larger for words that are unpredictable with respect to the context and therefore violate contextual expectations (Kutas and Hillyard 1984). This is because the context pre-activates (or leads people to predict) upcoming relevant features, thus a stimulus that is expected can be more easily integrated into its context.

Some emotion processing studies investigated the N400 in response to violations of an emotional context. In these studies, the preceding sentence stem provides a semantic and/or an emotional context which could be emotionally congruous or incongruous with the critical word. For instance, in the sentence "At the funeral I felt happy", "happy" constitutes a contextual violation or incongruency, since happiness is not an emotion that one would expect to feel in a funeral. Accordingly, these studies found larger N400s for inconsistent compared to consistent emotional target words (e.g., León et al. 2010). A few language studies also found a valence-specific effect on the N400. These studies reported reduced N400 effects for positive words compared to negative and neutral words in young adults (Martin-Loeches et al., 2012; Wang et al., 2013; Yao et al., 2016). Considering the functional significance of the $\mathrm{N} 400$, one interpretation of these findings is that higher expectations for positive information made positive words more easily integrated into the sentence context. In another line of research into the $\mathrm{N} 400$ and emotional processing, emotion violations were not operationalised as an incongruency between the emotional valence of the context and the critical word, but rather as an incongruency between the valence of the word or context 
and internal states or predispositions. For instance, positive judgements about oneself evoke reduced N400 effects compared to negative judgements, in line with the idea that most individuals associate information about the self with a positive valence (Watson et al. 2007; Zhou et al. 2013; Chen et al. 2014; Fields and Kuperberg 2015). Similarly, reduced N400 effects were found in participants with high mindfulness scores (Dorjee et al., 2015) and in participants who developed a positive mood following a mood-induction procedure, as opposed to participants with a negative mood (Chung et al., 1996).

The studies reviewed above thus suggest that the $\mathrm{N} 400$ is not just responsive to emotional incongruencies between a stimulus and its context, but also between a stimulus and the affective states or predispositions of the perceiver. This makes this ERP component an ideal candidate to examine the positivity effect since this effect should be associated with higher expectations for positive as opposed to negative information. To examine this possibility, in the current study we presented neutral sentence stems with negative, positive or neutral/semantically incongruent critical word ends. We hypothesised that young adults and older adults would display smaller N400s for positive end words-compared to neutral and negative ones-reflecting the higher expectation for those words. Note that the positivity effect results from a relative age difference between young and older participants in the processing of positive versus negative information (Reed, Chan and Mikels 2014). We therefore expected that in a group of older adults, the reduction of the N400 in response to positive information would be more pronounced compared to the group of younger participants.

To further examine the temporal dynamics of age-related differences in emotion processing, we also focused on later positive potentials. In language studies, positive deflections peaking between 500 and $800 \mathrm{~ms}$ with a centro-parietal distribution generally follow the N400. Different names have been attributed to this family of late positive components (Late Positive Potential, Late Positive Complex, P600; for a review, Ibanez et al. 2012). Here, we will refer to this component as Late Positive Complex (LPC), because this term is frequently used in studies of emotion processing. The LPC is generally enhanced 
for semantic and syntactic violations (Osterhout and Holcomb 1992; Kolk and Chwilla 2007) and low predictive words (Van Petten and Luka 2012). It is assumed that the LPC reflects reanalysis of the sentence, discourse-level repair and interpretation of semantically illconstructed language (Kuperberg 2007). There is a lack of consistency across studies in emotion-induced LPC effects, likely reflecting differences in task and stimuli used. For instance, the LPC was enhanced for positive words in some studies (Yao et al. 2016), and negative words in others (Holt, Lynn and Kuperberg, 2009; Dorjee et al. 2015); other studies have found a reduction (Espuny et al. 2018) or increase (Delaney-Busch and Kuperberg 2013) of this component for emotional stimuli in general, regardless of their valence, and others found no effects of emotions at all (Jimenez et al. 2012). Because of these conflicting findings, we could not formulate solid hypotheses on how the LPC would be affected by age and emotion. However, given its relevance for emotion processing, we included this component in our examination.

\section{METHODS}

\subsection{Participants}

We recruited 14 young and 19 older participants. We recruited a larger number of older participants based on the observation that signal-to-noise ratios are lower in older adults (Hämmerer et al. 2013) and participants in this group are more likely to be excluded from the sample at the analysis stage due to poor-quality EEG data. Young participants were university students with a mean age of 22 years ( $S D=3$ years; 9 females). Older adults had a mean age of 74 years (SD = 7 years, age range 64-88 years; 11 females) and 15 years of education on average (SD = 3 years). Participants were native English speakers and had normal or corrected-to-normal vision. With the exception of two older participants who reported mild use of selective serotonin reuptake inhibitors, no other participant reported current or recent use of psychotropic medication or sleeping aids. None of the participants reported a recent history of relevant psychiatric or neurological disease. Young participants were recruited at Kingston University London, whereas older participants were recruited from 
local senior centres situated in London. The experimental procedures were approved by the Kingston University Research Ethics Committee.

\subsection{Materials}

Sentences were constructed from 46 emotionally-neutral sentence stems. We generated three sentence versions from each sentence stem. The three versions were identical with the exception of the last, critical word, which attributed positive (e.g., "During the Christmas period people often feel joyful"), negative (e.g., "During the Christmas period people often feel helpless") or neutral (e.g., "During the Christmas period people often feel crunchy") meaning to the sentence. Sentence stems were not predictive of the critical word. In positive and negative sentences the critical word was always semantically congruent with the sentence stem, although we expected that the positivity bias would have resulted in a more difficult integration of negative critical words with the sentence context. In neutral sentences, the critical word was always semantically incongruent. This resulted in 138 sentences in total (46 positive, 46 negative, 46 neutral/incongruent). Twelve additional sentences (four for each valence condition) were presented before the start of the experiment to have participants familiarise with the stimulus material.

\subsection{Pre-test of the sentences used in the experiment}

Before data collection, sentences were pretested to an independent sample of 23 people (mean age 25, standard deviation 8 years, all females) to ensure that the sentences had positive, negative or neutral connotation. To this aim, participants were given a list of 296 sentences in an online questionnaire. The list consisted of identical neutral sentence stems with positive, negative, neutral/incongruent and neutral/congruent sentence endings. There were 74 randomly-intermixed sentence stems for each of the four valence conditions.

Participants were asked to indicate whether each sentence was positive, negative or neutral. If more than $50 \%$ of participants evaluated one sentence differently from their original connotation, the four versions of that sentence were not selected for the experiment. Most participants evaluated neutral/congruent sentence endings as either positive or negative, 
therefore this condition was deleted to avoid bias in the stimulus material. For the positive, negative and neutral/incongruent condition, the pre-test eliminated 24 sentences, resulting in the 150 sentences selected for the actual experiment (including 12 practice sentences). On average, the selected negative sentences were judged as negative by $89 \%$ of participants and the selected positive sentences were judged as positive by $86 \%$ of participants.

\subsection{Procedure}

Upon arrival to the lab, participants filled out the Mini Mental State Examination (MMSE, Folstein, Folstein and McHugh 1975) and the Geriatric Depression Scale (GDS, Yesavage et al. 1982). The MMSE and GDS were administered before the study phase to exclude from further testing i) participants with an MMSE score below 24, which is considered the cut-off for normal cognitive functioning (e.g., Fayers et al. 2005) and ii) a GDS score above 10, indicating moderate depression (Yesavage et al. 1982). None of the participants met these criteria, therefore all recruited participants completed the experiment. Participants were then fitted with the EEG cap and sat in front of a computer screen. Sentences were presented word-by-word at the centre of the screen. Each word was presented in white Helvetica on a grey background using the Cogent 2000 toolbox (http://www.vislab.ucl.ac.uk/cogent.php). Trials began with a 1000-ms fixation mark, then each word appeared for 200 ms followed by a 500-ms blank. This is considered a typical stimulus onset asynchrony in N400 studies (Wlotko and Federmeier 2015). The last word (critical word) was presented for $1000 \mathrm{ms,} \mathrm{and}$ the inter-sentence interval varied randomly between 4500 and $5000 \mathrm{~ms}$. The order of sentences was randomised anew for each participant. Twelve practice trials preceded the beginning of the experiment.

\subsection{EEG recording and pre-processing}

EEG was acquired with a 64-channel Biosemi Active Two system (BioSemi, Amsterdam, the Netherlands). Electrodes were positioned according to an equidistant montage using an elasticated cap (http://www.biosemi.com/pics/cap 64 layout medium.jpg). Scalp electrodes also included a Common Mode Sense active electrode and a Driven Right Leg passive 
electrode, which are customary used in Biosemi EEG systems to replace the ground electrode. Two additional electrodes were placed on the left and right mastoids, and four electrodes were used to record the electrooculogram (EOG): one above and one below the left eye for vertical EOG, and two on the outer canthus of each eye for horizontal EOG. EEG signals were digitised at $2048 \mathrm{~Hz}$ with a bandwidth of DC to $409 \mathrm{~Hz}$ using a fifth-order digital sinc filter. Electrode offsets were kept between $620 \mathrm{mV}$. Participants were asked to minimise blinking, movements and muscle contraction during the experiment. Offline analyses were conducted using EEGLAB (Delorme and Makeig 2004) and ERPLAB (Lopez-Calderon and Luck 2014). Data were digitally filtered between 0.1 and $30 \mathrm{~Hz}$. The EEG continuous recording was then segmented into epochs time-locked to the onset of the critical word. Epochs lasted from $100 \mathrm{~ms}$ prior to the onset of the critical word to $1200 \mathrm{~ms}$ afterwards. A 100-ms pre-stimulus baseline was applied. Ocular and muscular artifacts were corrected with independent component analysis (ICA; Delorme, Sejnowski, and Makeig 2007) and after ICA correction epochs were further scrutinised to remove artifacts still contaminating the data. Participants with less than 14 artifacts in the relevant conditions were excluded from further analyses (four older adults).

\subsection{ERP analysis}

ERP waveforms were computed by averaging artifact-free epochs surrounding the last, critical word as a function of the experimental condition (positive, negative or neutral/incongruent). In young adults, on average, ERP waveforms were based on 41.9 trials for positive, 41.2 trials for negative and 42.4 trials for neutral/incongruent sentences, respectively. In older adults, mean trial numbers were for 41.6 for positive, 42.3 for negative and 41.4 for neutral/incongruent sentences. In selected time windows (see Results), peak amplitudes and latencies of early ERP components and mean amplitudes of the N400 and LPC were extracted for 30 electrodes grouped in 10 regions of interest (ROI): Anterior Left AF7, F5, FC5; Anterior Central Left AF3, F3, FC3; Anterior Central AFz, Fz, FCz; Anterior Central Right AF4, F4, FC4; Anterior Right AF8, F6, FC6; Posterior Left PO7, P5, CP5; Posterior Central Left PO3, P3, CP3; Posterior Central POz, Pz, CPz; Posterior Central 
Right PO4, P4, CP4; Posterior Right PO8, P6, CP6. Due to marked differences in peak latencies between anterior and posterior electrodes, early ERP components were analysed separately for the 5 anterior and 5 posterior ROls. Peak amplitudes and latencies were submitted, separately for the anterior and posterior ROls, to a mixed model Analysis of Variance (ANOVA) with Age Group as a between-subjects factor (two levels: Young, Old), and the within-subjects factors Sentence Type (three levels: Positive, Negative, Neutral/Incongruent), ROI (five levels: Left, central left, central, central right, Right) and Electrode (three levels, each of the three electrodes comprising the ROI). For the N400 and LPC analysis, all 30 electrodes entered the analysis and mean amplitudes were submitted to a mixed model ANOVA with Age Group as a between-subjects factor (two levels: Young, Old), and the within-subjects factors Sentence Type (three levels: Positive, Negative, Neutral/Incongruent), Anterior-Posterior (two levels: Anterior, Posterior), ROI (five levels: Left, central left, central, central right, Right) and Electrode (three levels, each of the three electrodes comprising the ROI). For all ERP analyses reported in the results, the Greenhouse-Geisser correction (Greenhouse and Geisser 1959) was applied if violation of sphericity occurred, and in this case, the uncorrected degrees of freedom, the corrected $\mathrm{P}$ values, and the correction factor $\varepsilon$ are reported. The $\alpha$ level was set to 0.05 . Lower order interactions were not considered in the presence of higher order interactions and we only report significant results involving the factors Sentence Type and/or Age Group. The datasets generated during and/or analysed during the current study are available from the corresponding author on request.

\section{RESULTS}

\subsection{MMSE and GDS}

Mean scores for the MMSE were 27.53 in the older adults $(S D=1.78$, range $24-30)$ and 28.29 in young adults $(S D=0.99$, range $27-30)$. Mean scores for the GDS were 1.11 in older $(S D=1.48$, range $0-4)$ and $1.93(S D=1.33$, range $0-5)$ in young adults. There was no difference between the two groups in either the MMSE or the GDS scores ( $p s>0.05$ ). 


\subsection{Early ERP components}

At anterior sites, early ERP components in both groups consisted of a negativity peaking around $150 \mathrm{~ms}$ (N1) and a positivity peaking around $200 \mathrm{~ms}$ (P2). For statistical analyses, we measured the amplitude of the largest negative peak between 90 and $160 \mathrm{~ms}(\mathrm{~N} 1)$ and the largest positive peak between 160 and $250 \mathrm{~ms}$ (P2). These intervals were chosen on the basis of visual inspection and previous studies (Federmeier and Kutas 2005; Ding et al. 2016). The latency or amplitude of the N1 was unaffected by either Sentence Type or Age Group (all Ps>0.076). For P2 amplitudes, the interaction between Age Group and ROI was significant $\left(F_{4,108}=4.71, P=0.010, \eta^{2}=0.148, \varepsilon=0.561\right)$. Follow-up analyses in each $R O I$ indicated that P2 amplitudes were larger in younger adults in central ROIs (Anterior Central Left, $P=0.018$; Anterior Central, $P=0.001$; Anterior Central Right, $P=0.005$ ). The main effect of Sentence Type on the P2 latency $\left(F_{2,54}=4.22, P=0.020, \eta^{2}=0.135, \varepsilon=0.985\right)$ indicated that latencies were shorter for negative compared to positive sentence endings ( $P=0.008)$, however, neither positive nor negative sentence-endings differed from neutral/incongruent ones.

At posterior sites, early ERP deflections were characterised by a negative peak around $100 \mathrm{~ms}$ (N1), a positivity peaking around $150 \mathrm{~ms}$ (P2) and a negativity peaking around 200 ms (N2). Peak amplitudes and latencies were extracted in the 60-120 ms (N1),120-170 ms (P2) and 170-270 ms (N2) time windows. No effects involving Sentence Type or Group emerged for the N1 and P2 (all Ps>0.076). The amplitude of the N2 was larger for young than older adults in far Posterior Left electrodes $(P=0.005)$, as indicated by the significant interaction between Age Group and $\mathrm{ROI}\left(\mathrm{F}_{4,108}=5.75, \mathrm{P}=0.004, \eta^{2}=0.175, \varepsilon=0.537\right)$.

\subsection{N400}

Following the early ERP components, in young adults neutral/incongruent and negative sentence endings showed larger negativities compared to positive word ends. This effect appeared between 300 and 500 ms and was maximum at centro-posterior scalp sites (Figure 1 and 2). This is consistent with the latency and scalp distribution of the N400 (Kutas and Federmeier 2011). To analyse N400 effects, mean ERP amplitudes were extracted in 
the $300-500$ ms time window. This window was selected based on previous ERP studies (e.g. Kutas and Hillyard 1984; Fedrmeier and Kutas 2005; Fields and Kuperberg 2015) and upon inspection of the grand averaged ERPs. We found a significant interaction between Sentence Type, Anterior-Posterior and Age Group $\left(F_{2,54}=3.73, P=0.040, \eta^{2}=0.121, \varepsilon=0.815\right)$. This interaction was decomposed with separate follow-up ANOVAs in each group. In young adults, the interaction between Sentence Type and Anterior-Posterior was significant $\left(F_{2,26}=8.34, P=0.002, \eta^{2}=0.391\right)$. Main effects of Sentence Type emerged at anterior $\left.F_{2,26}=7.68, P=0.002, \eta^{2}=0.371\right)$ and posterior $\left(F_{2,26}=8.89, P=0.001, \eta^{2}=0.406\right)$ electrodes. At anterior electrodes, pairwise comparisons showed that negative sentence endings were more positive-going than positive ones $(\mathrm{P}=0.002)$. At posterior electrodes, positive sentence endings elicited smaller N400s than negative $(P<0.001)$ and neutral/incongruent ones $(P=0.017)$, which did not differ from one another $(P=0.270)$, Figure 1 and 2. Neither the main effect of Sentence Type nor the interaction between Sentence Type and Anterior-Posterior were significant in older adults $(P=0.476$ and $P=0.233$, respectively).

\subsection{LPC}

A positive peak followed the N400, peaking at around $600 \mathrm{~ms}$ at posterior scalp sites, which we identified as a LPC (Figure 1 and 2). The LPC was examined by extracting mean amplitudes in the 500-800 ms interval, as in previous studies (e.g., Ding et al. 2016). We found a significant interaction between Sentence Type, Anterior-Posterior and Age Group $\left(F_{2,54}=3.82, P=0.043, \eta^{2}=0.124, \varepsilon=0.725\right)$. This interaction was decomposed with separate follow-up ANOVAs in each group. There was no significant main effect of Sentence Type nor interaction between Sentence Type and Anterior-Posterior in young adults $(P=0.919$ and $P=0.222$, respectively). In older adults, the interaction between Sentence Type and AnteriorPosterior was significant $\left(\mathrm{F}_{2,28}=12.70, \mathrm{P}<0.001, \mathrm{\eta}^{2}=0.476\right)$. At anterior electrodes (main effect of Sentence Type, $\left.F_{2,28}=9.28, P=0.001, \eta^{2}=0.398\right)$ Bonferroni-corrected pairwise comparisons showed that neutral/incongruent sentence endings were more negative going than positive $(P=0.004)$ and negative ones $(P=0.023)$, which did not differ from one another $(P=0.999)$, Figure 1 and 2. At posterior electrodes (main effect of Sentence Type, 
$\left.F_{2,26}=12.90, P<0.001, \eta^{2}=0.479\right)$, the LPC was larger for neutral/incongruent sentence endings compared to than positive $(P=0.002)$ and negative ones $(P=0.003)$, which did not differ from one another $(\mathrm{P}=0.999)$.

\section{DISCUSSION}

In the current study we aimed to examine age-related differences in the temporal dynamics of emotion processing. In particular, we were interested in understanding whether the frequently reported preferential processing of positive information in old age would emerge at an early, automatic processing stage, or rather at a later, meaning-based stage. To achieve this goal, we used a language paradigm that allowed us to investigate early ERP components as well as later components, namely the N400 and the LPC.

At an early processing stage, the anterior P2 and posterior N2 amplitudes were larger in younger compared to older adults, as shown in previous work (Kemmer et al. 2004; Federmeier and Kutas 2005; Getzmann, Falkenstein, and Gajewski 2014). With the exception of shorter anterior P2 latencies in both groups for negative compared to positive sentence endings, neither of which differed from neutral/incongruent sentences, we did not find any other modulation of early ERP components by word valence. More importantly, we did not observe differences between young and older adults in early emotional processing and did not find any evidence of a positivity effect in either group. These results are at odds with previous studies showing a modulation of early ERP components as a function of emotional valence. It should be noted, however, that those studies generally used isolated words (Herbert et al. 2006; Kanske and Kotz 2007; Kissler and Herbert 2013; Wang et al. 2013) or faces (Pollock et al. 2012; Rellecke et al. 2012; Hilimire et al. 2014). In paradigms where ERPs are elicited in response to words embedded in sentence contexts, early ERP components may be less sensitive to emotional modulations, perhaps due to a facilitation of early lexical processing by top-down, contextual-driven mechanisms offered by the previous sentence stem. Furthermore, higher arousal and emotional saliency involved in face 
processing may explain an early emotional modulation of ERP components (Dehaene and Changeux 2011), which we did not observe in the current study.

Age differences in emotion processing were evident at later processing stages. In young adults, the N400 was larger for neutral/incongruent and negative sentence endings and reduced for positive sentence endings. A larger N400 for neutral/incongruent sentence endings was predictable considering that the N400 is generally larger for semantic anomalies or incongruencies and unexpected sentence endings (Kutas and Hillyard 1980, 1984). The pattern of results with smaller N400 effects for positive compared to both neutral/incongruent and negative words suggests different expectations for the upcoming critical word. We speculate that the positivity effect may have led young adults to predict positive sentence endings over negative ones, which were equally unexpected as neutral/incongruent words. In turn, this may have determined an easier semantic integration of positive endings into the context, and, as a consequence, smaller N400 amplitudes. This is consistent with previous findings that indicated better semantic integration of positive information compared with negative one (Kanske and Kotz 2007; Wang and Yao 2012) and previous research showing smaller N400s for positive information in young adults (MartínLoeches et al. 2012; Wang et al. 2013; Yao et al. 2016). Our findings are also consistent with the results of previous studies showing that the amplitude of the N400 in response to positive stimuli is smaller if there is an internal predisposition towards positively-valenced information, for instance, due to positive mood induction (Chung et al. 1996), mindfulness (Dorjee et al. 2015), or in response to positive judgements about oneself (Watson et al. 2007; Zhou et al. 2013; Chen et al. 2014; Fields and Kuperberg 2015). Altogether, the observation that positivity effects in older adults are evident in modulations of the $\mathrm{N} 400$ and not early ERP components suggest that the effect is associated with meaning-based processing rather than enhanced perceptual or attentional processing for positive information.

In older adults, we hypothesised smaller N400 effects for positive sentence endings compared to young adults as a consequence of the positivity effect in old age. Contrary to 
this expectation, we did not find any N400 effect in older adults—neutral/incongruent words did not differ from either emotional endings. We assume that this lack of N400 effects is due to fundamentally different processing engaged by the two groups, rather than by a difference in emotional processing per se or by a lack of positivity effects in older adults. One hypothesis is that older adults do not utilise contextual information to the same degree as younger adults in facilitating the processing of sentence endings. Older adults may have reduced abilities to use contextual information to predict upcoming information, making contextual integration upon the presentation of the sentence ending more demanding and shifting cognitive resources away from semantic elaboration at the N400 processing stage. Accordingly, previous studies have found smaller or absent N400 effects in older adults in response to semantic incongruencies (Federmeier and Kutas 2005; Payne and Federmeier 2018).

In older adults, ERP effects were evident at later stages of processing and took the form of enhanced amplitudes of the LPC for neutral/incongruent sentence endings compared to both positive and negative ones. The LPC is generally larger for semantically incongruent information (Ding et al., 2016) and is suggested to arise from deep evaluative processing and semantic retrieval (Kutas and Federmeier 2000), re-analysis (Van Petten and Luka 2012), or well-formedness check of the sentence (Szewczyk and Schriefers 2011). One idea to explain LPC effects in older adults is that individuals tried to compensate for the decreased semantic processing in the N400 time window, or they used the time window of the LPC to semantically reanalyse the sentence and the critical words. Alternatively, the effect could be due to differences in how emotional and neutral sentences are analysed at the semantic level at this stage of processing. In the absence of a neutral/congruent condition we cannot adjudicate between these two hypotheses, although the first one seems more plausible given the functional significance of the LPC. Interestingly, consistent with previous investigations (Xu et al. 2017), LPC effects did not emerge in young adults, perhaps because in this group any further semantic processing was not needed as the words were fully processed at the N400 stage. 
In older but not younger adults, a frontal positivity followed the N400 which showed larger amplitudes compared to either emotional words. Given its time of occurrence and scalp distribution, this effect could be related to a post-N400 positivity (PNP), which has been associated with detecting an incorrect prediction (Federmeier et al. 2007) or updating the current processing in light of unanticipated information (Van Petten and Luka 2012). Our results might thus indicate that older adults have used neural resources for context-updating processing (Dave et al. 2018). Why this waveform was more negative going for neutral/incongruent than emotional words is unclear though and since this component was not anticipated we do not feel confident to speculate upon it at present.

Although we did find a positivity effect in younger adults indexed by the N400, we did not find any evidence of positivity effects in older adults at any point in time. This contradicts our expectation of finding larger positivity effects in older compared to younger adults. On the one hand, we cannot rule out that in our group of older individuals there was no predisposition for positive information in general. However, older participants who took part in the experiment were healthy, active and non-depressed (as evidenced by GDS scores) individuals, therefore it is unlikely that they did not have the well-established preference for positive information. A number of studies have failed to detect a positivity effect in old age suggesting that the effect may depend on particular tasks or stimulus parameters (e.g., Denburg, Buchnann, Tranel and Adolphs 2003). We thus believe that, for the reasons outlined above, the cognitive processing at play in the current paradigm obscured any positivity effect in the ERP components under investigation.

Some limitations of the current investigation are worth mentioning, First, although we did have enough power to detect differences in both groups, our sample size was relatively small. Second, we did not measure baseline group differences in brain structure or function. Age-related changes in brain structure or function can complicate the interpretation of between-group comparisons of ERP waveforms and a frequent observation in ERP studies is that of a reduced amplitude of components or effects in older individuals. The results of the current investigation however cannot be explained by a reduction of ERP effects 
because the LPC effects were indeed larger in older adults. Similarly, we did not have a comprehensive measurement of cognitive functioning. Although the MMSE scores suggest that all participants in our sample of older adults were cognitively intact, these scores only provide a global indication of the cognitive status and they two groups may have differed in specific cognitive functions that are relevant for the performance of the task (e.g., working memory). Three caveats are also worth mentioning regarding the stimuli. We pretested the sentences in young adults only, therefore we cannot rule out that for some sentences the emotional valence was different in older adults. Furthermore, although we carefully selected the sentence stems to be as neutral as possible, some of them may have induced participants to expect a specific emotional content. Importantly, we acknowledge that a neutral/congruent condition would have been a better control condition to assess bias in emotion processing. The experiment was indeed planned with a neutral/congruent condition, but sentences belonging to this condition had to be eliminated at the pre-testing stage (see paragraph 2.2). As mentioned earlier, the lack of a neutral/congruent condition makes it difficult to disentangle valence from congruency effects in the LPC of older adults whereby both valence conditions differed from the neutral/incongruent condition.

To conclude, in the current investigation we showed an age-related difference in the temporal dynamics of emotion processing. Whereas younger adults showed easier processing of positive vs negative information, older adults did not show a bias towards either emotional valence. When processing emotional content, older adults seem to engage different cognitive strategies than their younger counterparts, possibly due to an increased effort in semantic processing, which lead to an overall reduction in emotion-specific processing. These findings further our understanding of emotional processing in older age and may suggest ways in which emotional disorders in older adults can emerge. 


\section{DECLARATIONS}

- The authors declare that the study has been approved by the Kingston University Ethics Committee and has been performed in accordance with the ethical standards laid down in the 1964 Declaration of Helsinki.

- All research participants gave their informed consent prior to their inclusion in the study

- The authors declare that they have no conflict of interest 


\section{REFERENCE LIST}

Carstensen LL (2006) The influence of a sense of time on human development. Science 312:1913-1915

Carstensen LL, Turan B, Scheibe S, Ram N, Ersner-Hershfield H, Samanez-Larkin GR, Nesselroade JR (2011) Emotional experience improves with age: Evidence based on over 10 years of experience sampling. Psychology and Aging 26:21-33.

https://doi.org/10.1037/a0021285

Charles ST, Mather M, Carstensen LL (2003) Aging and emotional memory: The forgettable nature of negative images for older adults. Journal of Experimental Psychology: General 132:310-324. https://doi.org/10.1037/0096-3445.132.2.310

Chen Y, Zhong Y, Zhou H, Zhang S, Tan Q, Fan W (2014) Evidence for implicit self-positivity bias: an event-related brain potential study. Experimental Brain Research 232:985-994. https://doi.org/10.1007/s00221-013-3810-z

Chung G, Tucker DM, West P, Potts G, Liotti M, Luu P, Hartry AL (1996) Emotional expectancy: Brain electrical activity associated with an emotional bias in interpreting life events. Psychophysiology 33:218-233. https://doi.org/10.1111/j.1469-8986.1996.tb00419.x

Craik F, Salthouse T (2002) The Handbook of Aging and Cognition, 3rd edn. Lawrence Erlbaum, Mahwah

Dave S, Brothers TA, Traxler MJ, Ferreira F, Henderson JM, Swaab TY (2018) Electrophysiological evidence for preserved primacy of lexical prediction in aging. Neuropsychologia 117:135-147. https://doi.org/10.1016/j.neuropsychologia.2018.05.023 Dehaene S, Changeux JP (2011) Experimental and theoretical approaches to conscious processing. Neuron 70:200-227. https://doi.org/10.1016/j.neuron.2011.03.018

Delaney-Busch N, Kuperberg G (2013) Friendly drug-dealers and terrifying puppies: affective primacy can attenuate the N400 effect in emotional discourse contexts. Cognitive, 
Affective, \& Behavioral Neuroscience 13:473-490. https://doi.org/10.3758/s13415-013-01595

Delorme A, Makeig S (2004) EEGLAB: an open source toolbox for analysis of single-trial EEG dynamics including independent component analysis. Journal of Neuroscience Methods 134:9-21. https://doi.org/10.1016/j.jneumeth.2003.10.009

Delorme A, Sejnowski T, Makeig S (2007) Enhanced detection of artifacts in EEG data using higher-order statistics and independent component analysis. Neuroimage 34:1443-1449-21. https://doi.org/10.1016/j.neuroimage.2006.11.004

Denburg NL, Buchanan TW, Tranel D, Adolphs R (2003) Evidence for preserved emotional memory in normal older persons. Emotion 3:239-253. https://doi.org/10.1037/15283542.3.3.239

De Pascalis V, Arwari B, D’Antuono L, Cacace I (2009) Impulsivity and semantic/emotional processing: An examination of the N400 wave. Clinical Neurophysiology 120:85-92. https://doi.org/10.1016/j.clinph.2008.10.008

Ding J, Wang L, Yang Y (2016) The dynamic influence of emotional words on sentence comprehension: An ERP study. Cognitive, Affective, \& Behavioral Neuroscience 16:433-446. https://doi.org/10.3758/s13415-016-0403-x

Dorjee D, Lally N, Darrall-Rew J, Thierry G (2015) Dispositional mindfulness and semantic integration of emotional words: Evidence from event-related brain potentials. Neuroscience Research 97:45-51. https://doi.org/10.1016/j.neures.2015.03.002

Espuny J, Jiménez-Ortega L et al (2018) Event-related brain potential correlates of words' emotional valence irrespective of arousal and type of task. Neuroscience Letters 670:83-88. https://doi.org/10.1016/j.neulet.2018.01.050

Fayers PM, Hjermstad MJ, Ranhoff AH, Kaasa S, Skogstad L, Klepstad P, Loge JH (2005) Which mini-mental state exam items can be used to screen for delirium and cognitive 
impairment? Journal of Pain and Symptom Management 30:41-50.

https://doi.org/10.1016/j.jpainsymman.2005.05.001

Federmeier KD, Kutas M (2005) Aging in context: age-related changes in context use during language comprehension. Psychophysiology 42:133-141. https://doi.org/10.1111/j.14698986.2005.00274.x

Federmeier KD, Wlotko EW, De Ochoa-Dewald E, Kutas M (2007) Multiple effects of sentential constraint on word processing. Brain Research 1146:75-84. https://doi.org/10.1016/j.brainres.2006.06.101

Fields EC, Kuperberg GR (2015) Loving yourself more than your neighbor: ERPs reveal online effects of a self-positivity bias. Social cognitive and affective neuroscience 10:12021209. https://doi.org/10.1093/scan/nsv004

Folstein MF, Folstein SE, McHugh PR. "Mini-mental state" (1975) A practical method for grading the cognitive state of patients for the clinician. J Psychiatr Res 12:189-98. https://doi.org/10.1016/0022-3956(75)90026-6

Galli G, Sirota M, Materassi M, Zaninotto F, Therry P (2017) Brain indices of disagreement with one's social values predict EU referendum voting behaviour. Social, Cognitive, and Affective Neuroscience 12:1758-1765. https://doi.org/10.1093/scan/nsx105

Getzmann S, Falkenstein M, Gajewski PD (2014) Neuro-behavioral correlates of postdeviance distraction in middle-aged and old adults. Journal of Psychophysiology 28:178-186 https://doi.org/10.1027/0269-8803/a000124

Greenhouse SW, Geisser S (1959) On methods in the analysis of profile data. Psychometrika 24:95-112. https://doi.org/10.1007/BF02289823

Hämmerer D, Li SC, Völkle M, Müller V, Lindenberger U (2013) A lifespan comparison of the reliability, test-retest stability, and signal-to-noise ratio of event-related potentials assessed 
during performance monitoring. Psychophysiology 50:111-23. https://doi.org/10.1111/j.14698986.2012.01476.x

Herbert C, Kissler J, Junghöfer M, Peyk P, Rockstroh B (2006) Processing of emotional adjectives: Evidence from startle EMG and ERPs. Psychophysiology 43:197-206. https://doi.org/10.1111/j.1469-8986.2006.00385.x

Hilimire MR, Mienaltowski A, Blanchard-Fields F, Corballis PM (2014) Age-related differences in event-related potentials for early visual processing of emotional faces. Social Cognitive \& Affective Neuroscience 9:969-76. https://doi.org/10.1093/scan/nst071

Holt DJ, Lynn SK, Kuperberg GR (2009) Neurophysiological correlates of comprehending emotional meaning in context. Journal of Cognitive Neuroscience 21:2245-2262. https://doi.org/10.1162/jocn.2008.21151

Ibanez A, Melloni M et al (2012) What event-related potentials (ERPs) bring to social neuroscience? Social Neuroscience 7:632-649.

https://doi.org/10.1080/17470919.2012.691078

Isaacowitz DM, Allard ES, Murphy NA, Schlangel M (2009) The time course of age-related preferences toward positive and negative stimuli. The journals of gerontology. Series B, Psychological sciences and social sciences 64:188192.https://doi.org/10.1093/geronb/gbn036 Isaacowitz DM, Choi Y (2011) The malleability of age-related positive gaze preferences: Training to change gaze and mood. Emotion 11:90-100. https://doi.org/10.1037/a0021551. Isaacowitz DM, Toner K, Goren D, Wilson HR (2008) Looking while unhappy: Moodcongruent gaze in young adults, positive gaze in older adults. Psychological Science 19:848853. https://doi.org/10.1111/j.1467-9280.2008.02167.x 
Jimenez-Ortega L, Martín-Loeches M et al (2012) How the emotional content of discourse affects language comprehension. PLoS One 7:e33718.

https://doi.org/10.1371/journal.pone.0033718

Kanske P, Kotz SA (2007) Concreteness in emotional words: ERP evidence from a hemifield study. Brain Research 1148:138-148. https://doi.org/10.1016/j.brainres.2007.02.044

Kemmer L, Coulson S, De Ochoa E, Kutas M (2004) Syntactic processing with aging: An event-related potential study. Psychophysiology 41:372-384. https://doi.org/10.1111/14698986.2004.00180.x

Kennedy Q, Mather M, Carstensen LL (2004) The role of motivation in the age-related positivity effect in autobiographical memory. Psychological Science 15:208-214.

https://doi.org/10.1111/j.0956-7976.2004.01503011.x

Kissler J, Herbert C (2013) Emotion, Etmnooi, or Emitoon? Faster lexical access to emotional than to neutral words during reading. Biological Psychology 92:464-79. https://doi.org/10.1016/j.biopsycho.2012.09.004

Kolk H, Chwilla D (2007) Late positivities in unusual situations. Brain and Language 100:257-261. https://doi.org/10.1016/j.bandl.2006.07.006

Kuperberg GR (2007) Neural mechanisms of language comprehension: Challenges to syntax. Brain Research 1146:23-49. https://doi.org/10.1016/j.brainres.2006.12.063

Kutas M, Federmeier KD (2000) Electrophysiology reveals semantic memory use in language comprehension. Trends in Cognitive Sciences 4:463-470. https://doi.org/10.1016/S1364-6613(00)01560-6

Kutas M, Federmeier KD (2011) Thirty years and counting: finding meaning in the N400 component of the event-related brain potential (ERP). Annual Review of Psychology 62:621647. https://doi.org/10.1146/annurev.psych.093008.131123 
Kutas M, Hillyard SA (1980) Reading senseless sentences: brain potentials reflect semantic incongruity. Science 207:203-5. https://doi.org/10.1126/science.7350657

Kutas M, Hillyard SA (1984) Brain potentials during reading reflect word expectancy and semantic association. Nature 307:161-3. http://dx.doi.org/10.1038/307161a0

León I, Díaz JM, de Vega M, Hernández JA (2010) Discourse-based emotional consistency modulates early and middle components of event-related potentials. Emotion 10:863-873. https://doi.org/10.1037/a0019983

Löckenhoff CE, Carstensen LL (2007) Aging, emotion, and health-related decision strategies: Motivational manipulations can reduce age differences. Psychology and Aging 22:134-146. https://doi.org/10.1037/0882-7974.22.1.134

Lopez-Calderon J, Luck SJ (2014) ERPLAB: an open-source toolbox for the analysis of event-related potentials. Frontiers In Human Neuroscience 8:213. https://doi.org/10.3389/fnhum.2014.00213

Luo L, Craik FI (2008) Aging and memory: a cognitive approach. Canadian Journal of Psychiatry 53:346-353. https://doi.org/10.1177/070674370805300603

Martín-Loeches M, Fernández A et al (2012) The influence of emotional words on sentence processing: Electrophysiological and behavioral evidence. Neuropsychologia 50:3262-3272. https://doi.org/10.1016/j.neuropsychologia.2012.09.010

Mather M, Carstensen LL (2003) Aging and attentional biases for emotional faces. Psychological Science 14:409-415. https://doi.org/10.1111/1467-9280.01455 Mikels JA, Larkin GR, Reuter-Lorenz PA, Carstensen LL (2005) Divergent trajectories in the aging mind: Changes in working memory for affective versus visual information with age. Psychology and Aging 20:542-553. https://doi.org/10.1037/0882-7974.20.4.542 
Noh SR, Lohani M, Isaacowitz DM (2011) Deliberate real-time mood regulation in adulthood: The importance of age, fixation and attentional functioning. Cognition and Emotion 25:9981013. https://doi.org/10.1080/02699931.2010.541668.

Osterhout L, Holcomb PJ (1992) Event-related brain potentials elicited by syntactic anomaly. Journal of memory and language 31:785-806. https://doi.org/10.1016/0749-596X(92)90039Z

Payne BR, Federmeier KD (2018) Contextual constraints on lexico-semantic processing in aging: Evidence from single-word event-related brain potentials. Brain Research 1687:117128. https://doi.org/10.1016/j.brainres.2018.02.021

Pollock JW, Khoja N, Kaut KP, Lien MC, Allen P (2012) Electrophysiological evidence for adult age related sparing and decrements in emotion perception and attention. Frontiers in Integrative Neuroscience 6:1-14. https://doi.org/10.3389/fnint.2012.00060

Reed AE, Chan L, Mikels JA (2014) Meta-analysis of the age-related positivity effect: age differences in preferences for positive over negative information. Psychology and aging 29:115. https://doi.org/10.1037/a0035194

Rellecke J, Sommer W, Schacht A (2012) Does processing of emotional facial expressions depend on intention? Time-resolved evidence from event-related brain potentials. Biological Psychology 90:23-32. https://doi.org/10.1016/j.biopsycho.2012.02.002

Szewczyk JM, Schriefers H (2011) Is animacy special?: ERP correlates of semantic violations and animacy violations in sentence processing. Brain Research 1368:208-221. https://doi.org/10.1016/j.brainres.2010.10.070

Urry HL, Gross JJ (2010) Emotion regulation in older age. Current Directions in Psychological Science 19:352-357 https://doi.org/10.1177/0963721410388395 
Van Berkum JJ, Holleman B, Nieuwland M, Otten M, Murre J (2009) Right or wrong? The brain's fast response to morally objectionable statements. Psychological Science 20:1092-9. https://doi.org/10.1111/j.1467-9280.2009.02411.x

Van Petten C, Luka BJ (2012) Prediction during language comprehension: Benefits, costs, and ERP components. International Journal of Psychophysiology 83:176-190. https://doi.org/10.1016/j.ijpsycho.2011.09.015

Wang L, Bastiaansen M, Yang Y, Hagoort P (2013) ERP evidence on the interaction between information structure and emotional salience of words. Cognitive, Affective, \& Behavioral Neuroscience 13:297-310. https://doi.org/10.3758/s13415-012-0146-2

Wang ZH, Yao Z (2012) Concreteness effects of emotional noun words: evidences from ERP. Acta Psychologica Sinica 44:154-165

Watson LA, Dritschel B, Obonsawin MC, Jentzsch I (2007) Seeing yourself in a positive light: brain correlates of the self-positivity bias. Brain Research 1152:106-110. https://doi.org/10.1016/j.brainres.2007.03.049

Wlotko EW, Federmeier KD (2015) Time for prediction? The effect of presentation rate on predictive sentence comprehension during word-by-word reading. Cortex 68:20-32. https://doi.org/10.1016/j.cortex.2015.03.014 Xu N, Hou X et al (2017) Age-related temporal-spatial dynamic ERP changes during sentence comprehension. Neuroscience Letters 645:74-79. https://doi.org/10.1016/j.neulet.2017.02.074 Yao Z, Wang Z (2014) Concreteness of positive word contributions to affective priming: An ERP study. International Journal of Psychophysiology 93:275-282. https://doi.org/10.1016/j.jpsycho.2014.06.005

Yao Z, Yu D, Wang L, Zhu X, Guo J, Wang Z (2016) Effects of valence and arousal on emotional word processing are modulated by concreteness: Behavioral and ERP evidence 
from a lexical decision task. International Journal of Psychophysiology 110:231-242.

https://doi.org/10.1016/j.jpsycho.2016.07.499

Yesavage JA, Brink TL, Rose TL et al (1982) Development and validation of a geriatric depression screening scale: a preliminary report. Journal of Psychiatry Research 17:37-49.

https://doi.org/10.1016/0022-3956(82)90033-4

Zhou A, Li S et al (2013) Perspective taking modulates positivity bias in self-appraisals:

Behavioral and event-related potential evidence. Social Neuroscience 8:326-333.

https://doi.org/10.1080/17470919.2013.807873 


\section{FIGURE LEGENDS}

Figure 1. ERP waveforms at three representative frontal electrodes and three representative posterior electrodes. The analysis window of the N400 is highlighted in yellow (300-500 ms). In this time window, waveforms for positive sentence endings were reduced in young adults. The analysis interval of the LPC is highlighted in grey $(500-800 \mathrm{~ms})$. In this time window, at posterior electrodes the LPC was larger for neutral/incongruent sentence endings in the elderly.

Figure 2. On the left, scalp distribution of the observed ERP difference between neutral and positive sentence endings (left) and neutral and negative sentence endings (right) in the two age groups. On the right, ERP waveforms at the POz electrode, where some of the effects were larger. The yellow box indicates the N400 analysis window (300-500 ms), and the grey box the LPC analysis window (500-800 ms). 